Aging Ment Health. 2017 October ; 21(10): 1065-1071. doi:10.1080/13607863.2016.1191060.

\title{
Living alone and depression: the modifying role of the perceived neighborhood environment
}

\author{
Sarah T. Stahl ${ }^{\mathrm{a}}$, Scott R. Beach ${ }^{\mathrm{b}}$, Donald Musa ${ }^{\mathrm{b}}$, and Richard Schulz ${ }^{\mathrm{a}, \mathrm{b}}$ \\ aDepartment of Psychiatry, University of Pittsburgh, Pittsburgh, PA, USA \\ bUniversity Center for Social and Urban Research, University of Pittsburgh, Pittsburgh, PA, USA
}

\begin{abstract}
Objective-Older adults who live alone are more likely to report feelings of depression than those who live with a spouse or other family members. This study examines the effects of residential status and perceived neighborhood characteristics on depression in middle-aged and older adults.
\end{abstract}

Methods-This study is based on a probability sample of 1049 adults aged 55-98 years ( $M=69$ years) residing in Allegheny County, Pittsburgh, PA, USA in 2014. Thirty percent of participants reported living alone. We tested a multivariate model using living alone (vs. living with a family member or others) and perceived neighborhood physical and social quality as predictors of depressive symptomatology while controlling for age, sex, race, education, and disability.

Results-Living alone (compared to living with a family member) was associated with elevated levels of depressive symptomatology. However, perceptions of neighborhood social quality moderated this association. Living alone was more highly associated with depression when the perceived social quality of the neighborhood was low. Neighborhood social quality was not associated with depression among older adults who lived with a family member. Perceptions of neighborhood physical quality were not significantly associated with depression.

Conclusions-Perceptions of good neighborhood social quality is important for adults who live alone, in terms of fewer symptoms of depression.

\section{Keywords}

Living alone; elderly; depression; neighborhood; environment

\section{Objective}

A substantial proportion of older adults live alone in the United States. In 2010, about 30\% (11 million) of older non-institutionalized persons lived alone. The likelihood of living alone is higher for older women (37\%) than men (19\%) and is very high among widowed elders (70\%). The proportion of adults living alone increases with advancing age; almost half

Correspondence to: Sarah T. Stahl.

Disclosure statement

No potential conflict of interest was reported by the authors. 
(47\%) of women aged 75 years and older live alone. Given the aging baby boom cohort, continued growth in the number of older adults who live alone is expected (Ortman, Velkoff, \& Hogan, 2014).

Living alone poses serious challenges for older adults. Older adults who live alone are more likely to report feelings of depression than those who live with a spouse or family member (Dean, Kolody, Wood, \& Matt, 1992; Xiu-Ying, Qian, Xiao-Dong, Xue-Mei, \& ChangQuan, 2012). They are also at high risk for early mortality (Holt-Lunstad, Smith, Baker, Harris, \& Stephenson, 2015). There are several reasons why living alone is associated with depression. First, living alone increases the risk for social isolation, especially among those who have no children or live far away from them. Individuals who are socially isolated receive significantly less emotional and instrumental support than those who are not socially isolated, which in turn increases the risk for depression (Cacioppo \& Hawkley, 2003). Older men who live alone are at higher risk for social isolation because they have a harder time maintaining social relationships than older women during late life. Second, adults who live alone are more likely to be poor and economically vulnerable, which also increases the risk of depression (Chou, Ho, \& Chi, 2006).

A question that remains unexplored in this area of research is whether the effect of living alone on depression varies according to particular neighborhood characteristics. There is a growing body of literature that demonstrates strong links between neighborhood characteristics and mental health and well-being in older adults (Cramm \& Nieboer, 2015; Elliott, Gale, Parsons, \& Kuh, 2014; Lawton, Nahemow, \& Yeh, 1980; Truong \& Ma, 2006; Yen, Michael, \& Perdue, 2009). For example, accessibility to resources in one's neighborhood (Lawton et al., 1980), perceived neighborhood safety (Roh et al., 2011), socioeconomic composition (Yen et al., 2009), and the built environment (accessible sidewalks, green spaces; Truong \& Ma, 2006) are all associated with good mental health. In addition to the physical aspect of one's neighborhood, feelings of neighborhood belonging, and social cohesion are also associated with good mental health (Elliott et al., 2014). Understanding the link between neighborhood characteristics and depression for older adults who live alone is important, given the desire for adults to age in place - that is, to remain in their own home and community for as long as possible (American Association of Retired Persons [AARP], 2011). The purpose of this study is to examine how the effect of living alone on depression may depend on older adults' perceptions of their neighborhood's physical and social environment.

In studies of older adults who live alone, predictors of depression mainly focus on individual demographic, physical, and mental health characteristics and often ignore the effects of the neighborhood context within which older adults live (La Gory \& Fitzpatrick, 1992). Neighborhood effects on depression vary by the personal traits of individuals and may be especially strong among adults who live alone (Chen \& Chen, 2015; Weden, Carpiano, \& Robert, 2008). For example, older adults who live alone may spend more time in their immediate residential neighborhood and, therefore, may be more dependent on their neighborhood for services, amenities, and social interactions than older adults who live with a spouse or family member (Kim \& Lee, 2015; Diez Roux, Borrell, Haan, Jackson, \& Schulz, 2004). Older adults who live alone may be especially sensitive to the health 
damaging (poor pavement conditions and heavy traffic) or health promoting (green spaces and accessible health care) aspects of their neighborhood's physical quality. Features of the neighborhood such as a sense of belonging with one's neighbors may influence the quantity and quality of the social support received from those interactions (Diez Roux et al., 2004). Neighborhood social cohesion, or a sense of belongingness with one's neighbors, is also associated with good physical and mental well-being, especially among the oldest old (Cramm \& Nieboer, 2015; Elliott et al., 2014).

Our study had two main objectives. First, we examined the effect of one's residential status (living alone vs. living with a family member) on depressive symptomatology. We then tested the extent to which the effect of residential status on depression may be moderated by older adults' perceptions of their neighborhoods' physical (accessible parks/green space) and social (close-knit neighbors) environment, controlling for individual characteristics known to be associated with depression. We hypothesize that a good physical neighborhood and good social quality of the neighborhood each provide an environment that buffers the effect of living alone on depression. Second, to further explore the living alone-depression link, we examined the modifying effect of neighborhood characteristics in single/never married, divorced, and widowed adults. We were particularly interested in whether neighborhood variables varied by marital status and were more (or less) important to different groups of adults who lived alone.

\section{Methods}

\section{Procedures}

The University Center for Social and Urban Research (UCSUR) at the University of Pittsburgh conducted a telephone survey using random digit dialing (RDD) sampling of both landline and cellular telephones with screening for adults aged 55 years and older. Landline RDD allowed for the over-sampling of African-Americans, by linking area codes/telephone exchanges to recent census data. Data were collected in UCSUR's computer-assisted telephone interviewing lab by trained interviewers in early 2014. Interviews took approximately 60 minutes to complete. The screening rate is actually the $23 \%$ of households that we were able to successfully screen for the presence of adults aged 55 years and older. The majority of the remaining $77 \%$ could not be contacted for screening or refused to be screened. Once we knew the household contained an adult 55+, we were able to complete interviews with $68 \%$ of these households (32\% of these refused the interview). The actual overall response rate was $0.23 \times 0.68=15.6 \%$ - low but typical for RDD surveys in 2014 . The survey asked about a broad range of topics related to quality of life for older adults in the region.

\section{Sample}

The survey targeted non-institutionalized, English-speaking adults aged 55 years and older living in Allegheny County, PA, USA. Allegheny County, which includes the City of Pittsburgh, has a population of over 1.2 million (2010 census data), with $13.3 \%$ of the population age 55-64 and $16.8 \%$ age 65 and over. Eighty-two percent of the population is White and the primary minority group is African-American at $13.2 \%$. The goal was to 
oversample African-Americans at $25 \%$ and also to have sufficient cases of adults younger than 65 for comparison, as this age group (55-64) makes up nearly $45 \%$ of the total population over age 55 in Allegheny County. The survey sample consisted of 397 adults aged 55-64, 359 adults aged 65-74, and 288 adults aged 75 plus (total $N=1049$ ); and 24\% $(n=254)$ of the sample was African-American. The sample slightly under-represents the 55-64 age group and over-represents the 65 and over age group. For this study, six participants did not report their living arrangements and were excluded from analyses.

Each case was statistically weighted to adjust for probability of selection. In addition, a poststratification weight was calculated using sex, age, race, education, employment status, and living arrangements to adjust for differential nonresponse across sub-groups. The probabilities of selection and post-stratification weights were combined to produce a final weight, which was used for all estimates in our analyses. This allowed us to draw inferences to the larger defined population of middle-aged and older adults in Allegheny County. The unweighted sample consisted of $28 \%$ men and $25 \%$ African-American; $32 \%$ earned a bachelor's degree or higher, $29 \%$ were working, $51 \%$ lived alone, $4 \%$ needed assistance with activities of daily living (ADLs), and 13\% needed assistance with instrumental activities of daily living (IADLs). A comparison of unweighted data with census data found that the interviewed sample was older, with a higher percentage of females, had a higher level of education, and was less likely to be working, but more likely to be living alone than was the general population of adults aged 55 and over living in the County. The final weight adjusted for these differences, and the weighted sample consisted of $44 \%$ men and $11 \%$ AfricanAmerican; 28\% earned a bachelor's degree or higher, 35\% were working, $30 \%$ lived alone, $3 \%$ needed assistance with ADLs, and $11 \%$ needed assistance with IADLs.

\section{Measures}

Depressive symptoms-Our main outcome variable was the frequency of depressive symptoms over the last two weeks, measured by the Patient Health Questionnaire (PHQ-8; Kroenke et al., 2009). Responses to the eight items ranged from 0 (not at all) to 3 (nearly every day) on a Likert-type scale and scored such that higher scores indicated a greater frequency of depressive symptoms. A total score of 10 or greater is associated with major depressive disorder (Kroenke et al., 2009). Depression was analyzed as a continuous variable.

Correlates of depressive symptoms-We controlled for variables known to be associated with depression in older adults including age, sex, race, education, and disability. Age was analyzed as a continuous variable. Race was categorized as White and Black/other. Education was characterized by 10 levels: eighth grade or less, some high school, high school graduate/general education development (GED), some college, associate's degree (occupational), associate's degree (academic), bachelor's degree, master's degree, professional degree, and doctoral degree. Education was analyzed as a continuous variable. The assessment of disability was divided into two levels, from the basic ADLs to the more advanced IADLs. There was a single question for each: 'Do you need help of other persons with your personal care needs, such as eating, bathing, dressing, toileting or moving from one place to another?' and 'Do you need the help of other persons with your routine care 
needs such as everyday household chores, managing money, taking medications, shopping, or transportation outside the house?' Both ADLs and IADLs were categorized as yes or no (i.e. reporting any ADL/IADL difficulties versus none).

Living arrangements-To determine residential status, participants were asked 'What are your current living arrangements?' Possible answers were (1) I live alone and (2) I live in a household with family or others. Marital status was analyzed using three categories: single/ never married, divorced, and widowed.

Neighborhood variables-Survey questions for neighborhood physical quality asked the respondent to consider the neighborhood and community in which he or she lived. Three items asked about the built environment including the physical condition of surrounding buildings, whether their neighborhood was a good place to walk/be physically active, and a good place for older adults to live. Responses to these items ranged from 1 (excellent) to 5 (poor). Three items asked about accessibility of grocery stores that sell healthy food, green spaces/public parks, and the convenience of the location of their current residence.

Responses to these items ranged from 1 (very convenient) to 4 (very inconvenient). One item asked about neighborhood safety. Reponses to this item ranged from 1 (very safe) to 4 (very unsafe). These items are not a part of a formal questionnaire, but are similar to items that neighborhood surveys typically use. We used linear transformation to convert the 5-point Likert scales to 4-point Likert scales. All seven items were averaged and interpreted such that lower scores indicate a better perception of the physical quality of one's neighborhood. The coefficient alpha for the physical quality scale was 0.80 .

Survey questions for the social quality of the neighborhood asked the respondent to think about his or her interactions with neighbors. Three items asked whether the people in their neighborhood are willing to help each other, can be trusted, and are close-knit. These items are a part of a widely used assessment of neighborhood social cohesion and trust (Sampson, Raudenbush, \& Earls, 1997). We added two additional items that asked about interactions with neighbors including the number of neighbors that respondents knew and how often respondents talked or visited with immediate neighbors. These five items all formed the same factor in a factor analysis that was run on seven possible survey items (that allowed for pair-wise calculation of the correlation matrix) that asked respondents about the social quality of their neighborhood. (The two items that did not load onto the social quality factor asked whether the people in respondents' neighborhood share the same values and get along with each other.) Reponses to all items ranged from 1 (strongly agree) to 4 (strongly disagree). All five items were averaged and interpreted such that lower scores indicate a better perception of the social quality of one's neighborhood. The coefficient alpha for the social quality scale was 0.76 .

\section{Statistical analyses}

We compared demographic, neighborhood, and depression variables between those who lived alone and those who lived with a family member using the $t$-test for continuous variables and the chi-square statistics for categorical variables. We also compared these variables across the three marital status groups. For our main analysis, we tested a 
multivariate regression model using living alone (vs. living with a family member), age, sex, race, education, ADLs, IADLs, neighborhood physical quality, and neighborhood social quality as predictors of depressive symptomatology. In the second step of our regression model, we added interaction terms separately to our model to determine if neighborhood physical quality and neighborhood social quality moderated the association between living alone and depressive symptoms. For our second research question, we created three dummy variables that captured the marital status of older adults who lived alone: single/never married $(n=69[22 \%])$, divorced $(n=77[25 \%])$, and widowed $(n=157[51 \%])$ (the remaining sample members, including those who were married and/or lived with a family member served as the referent category $[n=740])$. These dummy variables were entered as predictors in our regression model that also controlled for age, sex, race, education, ADLs, and IADLs. For all analyses, $p$ values smaller than 0.05 were considered to be statistically significant. All analyses were performed using SPSS, version 23.0 and STATA, version 12.

It is important to note that 43 participants (4\%) had missing data on one or more questions that asked about neighborhood social quality, and 29 participants (3\%) had missing data on one or more of the questions that asked about neighborhood physical quality. To preserve the full data set, we used all valid (non-missing) values necessary when constructing the mean scale. This strategy implicitly weights missing items with the overall mean. We compared the results of this approach with one using the observed data only, and with each of the two models, the results were identical.

\section{Results}

\section{Descriptive statistics}

Table 1 presents descriptive information for the 1043 adults at the time of the telephone survey. Based on the weighted estimates from the survey, the mean age of the respondents was 69 years ( $\mathrm{SD}=10$ years). The total sample was $44 \%$ men, $11 \%$ African-American, and $38 \%$ earned a bachelor's degree or higher. Thirty percent of adults reported living alone. Several group differences emerged for demographic, neighborhood, and depression variables between adults who lived alone and those who lived with a family member (zero adults reported having 'other living arrangements'). Adults who lived alone were older; they had a higher percentage of women and Black participants compared to adults who lived with a family member. They also reported needing assistance with IADLs compared to adults who lived with a family member. Adults who lived alone perceived their neighborhood physical quality to be significantly poorer than adults who lived with a family member. As expected, those who lived alone reported significantly more depressive symptoms than those who lived with a family member. Table 2 presents descriptive information across marital status groups. Single, divorced, and widowed adults all reported a poorer perception of their neighborhood's physical quality and the social quality of their neighborhood than adults who lived with a family member. Single, divorced, and widowed adults all reported a greater frequency of depressive symptoms than adults who lived with a family member. 


\section{Living alone and depressive symptomatology}

Table 3 shows the multiple regression model that tested the relationship between living alone and neighborhood characteristics on depressive symptomatology. Results indicate that younger age, less education, needing assistance with IADLs, and poor perception of one's social quality of neighborhood were all associated with increased depressive symptoms. Living alone (vs. living with a family member) was also associated with increased depressive symptoms, but this main effect was qualified by significant interaction between living alone and the social quality of the neighborhood. All other main effects including participant sex, race, ADLs, and neighborhood physical quality did not reach significance. The living alone $\times$ neighborhood physical quality interaction also did not reach significance $(p=0.34)$. Our final model accounted for a modest proportion of variance in depressive symptomatology $(16 \%)$.

To further understand the significant interaction, simple slopes of living alone (vs. living with a family member) on depression for high and low levels of neighborhood social quality were examined (Figure 1). Results indicate that living alone was significantly associated with depression only among those who perceived a poor social quality of their neighborhood, $\beta=0.20, p<0.001$. Living alone was not significantly associated with depression among those who perceived a good social quality of the neighborhood, $a=$ $-0.01, p=0.38$. Compared to those who lived with a family member, those who lived alone had higher levels of depressive symptomatology, but only among those who perceived a poor social quality of the neighborhood.

\section{Marital status and depressive symptomatology}

To further explore the living alone-depression link, we compared depressive symptomatology across three marital status groups in adults who lived alone. To test the role of marital status group in predicting depression, we constructed three dummy variables that captured the marital status of older adults who lived alone (adults who were married and/or lived with a family member served as the referent category). These variables were entered as predictors in our regression model that also controlled for socio-demographic variables (age, sex, race, and education) and disability (ADLs and IADLs). Results indicate that living alone and being single $(B=1.54, \mathrm{SE}(B)=0.76 ; p<0.01)$ and living alone and being divorced $(B=2.52$, SE $(B)=0.56 ; p<0.01)$ were each associated with increased depressive symptoms. Widowed adults who lived alone did not have significantly higher depressive symptoms than adults who lived with a family member. We then combined single and divorced adults into one group and tested the interaction with the social quality of the neighborhood. The interaction term was not significant $(p=0.07)$.

\section{Discussion}

This study investigated the extent to which the association between living alone and depressive symptomatology is moderated by neighborhood characteristics in a probability sample of middle-aged and older adults in Allegheny County, Pittsburgh PA, USA. We report three main findings. First, we observed an independent effect of one's living arrangement on depression, such that living alone (versus living with a family member) is 
associated with elevated levels of depressive symptomatology. This finding is consistent with many other studies (Chou et al., 2006; Dean et al., 1992; Fukunaga et al., 2012; XiuYing et al., 2012). Second, we found that neighborhood social quality modified the association between living alone and depression. Living alone was more highly associated with depression when the perceived social quality of the neighborhood was low.

Neighborhood social quality was not associated with depression among older adults who lived with a family member. Third, we found that specific relational components of living alone were more strongly related to depression. Those who lived alone and were single or divorced reported higher levels of depression than adults who lived with a family member. This finding is consistent with studies showing that marital status is an important correlation of depression (Kim \& McKenry, 2002). However, the modifying role of good neighborhood social quality was not more (or less) important to different groups of adults who live alone. Good neighborhood social quality was associated with less depressive symptomatology, regardless of adults' marital status. Finally, we did not find evidence that neighborhood physical quality was related to depression, as other work has suggested (Truong \& Ma, 2006).

There are several possible mechanisms through which neighborhood social quality might modify the living alone-depression relationship. Good neighborhood social quality may enhance instrumental support behaviors among neighbors including help with household tasks and transportation (Cramm \& Nieboer, 2015). Receiving practical help from neighbors may alleviate the stress associated with living alone (Robinette, Charles, Mogle, \& Almeida, 2013). Good neighborhood social quality may also increase the availability of social activities. Participating in social activities allows elders who live alone the opportunity to meet new people and develop social networks which may decrease feelings of loneliness and depression (Elliot et al., 2014). Good neighborhood social quality may also make elders feel safer or more secure, which may decrease feelings of depression. Finally, good neighborhood social quality may increase engagement in healthy behaviors associated with good mental health such as walking to visit neighbors.

What promotes feelings of neighborhood social cohesion? Our findings suggest that it is important for older adults who live alone to perceive the people in their neighborhood as close-knit and willing to help their neighbors. Other factors may play a role in shaping these perceptions and deserve to be studied in future research. For example, the physical environment can play a role in promoting feelings of social cohesion by affording contexts (senior centers and parks) for getting together and building a sense of belonging. Residents may also perceive good social cohesion because their neighborhood has an infrastructure (routine member meetings and social events) that supports older adults' social engagement in their communities. We also need a better understanding of how older individuals define their neighborhood. Some adults may identify with an entire suburb, several neighboring blocks, or with the houses that immediately surround them on the same street (Elliot et al., 2014). The measure of social quality used in this study focused on adults' immediate surroundings (interactions with neighbors). Those who define their neighborhood as several blocks might benefit from increasing locally organized groups while those who define their neighborhood as the houses that immediately surround them might benefit from strengthening relationships with neighbors. 
This study had several limitations. All of the data collected were cross-sectional making it difficult to make causal inferences. It is possible that those who are depressed are more likely to live alone. It is also possible that those who are depressed are more likely to perceive their neighborhood environment negatively (Chen \& Chen, 2015; Weden et al., 2008). Another limitation is the possibility of same-source bias, that is, the possibility that a third unobserved factor (e.g. physical activity levels) influenced adults' self-reports of both their neighborhood environment and mental health (Diez-Roux, 2007). Second, although we adjusted for an array of individual characteristics known to be associated with depressive symptoms in older adults, we did not have information on whether participants had a history of major depression. We also did not have enough information on adults' income to include it as an indicator for socioeconomic status (SES), although we did include education as an imperfect proxy for SES. Greater income may allow adults who live alone the opportunity to afford resources that promote their mental health. A third limitation relates to the subjective measurement of neighborhood physical quality. It is possible that objective neighborhood assessments (census tract data and neighborhood poverty/SES) are better predictors of depression than subjective assessments of the physical quality of one's neighborhood. This may explain why we did not find a significant relation between neighborhood physical quality and depression. However, some studies have suggested that census tracts may not reflect meaningful neighborhood boundaries (Macintyre, Maciver, \& Sooman, 1993). Future studies should use a mixed-methods approach to determine how subjective measures of the physical neighborhood relate to objective assessments of the built environment.

By considering the environmental context in which older adults live, this study highlights the need to consider social interactions with neighbors among older adults who live alone. Our data suggest that neighborhood social quality modifies the effect of living alone on depressive symptomatology. More research is needed to determine if environmentally driven interventions in the form of community development or social programs can significantly decrease depression by promoting feelings of neighborhood social cohesion.

\section{Acknowledgments}

Funding

Preparation of this manuscript was supported in part by grants from NIH [grant number P30 MH090333], [grant number K01 MH103467].

\section{References}

American Association of Retired Persons (AARP). Aging in place: A stat survey of livability policies and practices. 2011. Retrieved from https://assets.aarp.org/rgcenter/ppi/liv-com/aging-inplace-2011-full.pdf

Cacioppo JT, Hawkley LC. Social isolation and health, with an emphasis on underlying mechanisms. Perspectives in Biology \& Medicine. 2003; 46:39-52.

Chen J, Chen S. Mental health effects of perceived living environment and neighborhood safety in urbanizing China. Habitat International. 2015; 46:101-110.

Chou KL, Ho AH, Chi I. Living alone and depression in Chinese older adults. Aging \& Mental Health. 2006; 10(6):583-591. [PubMed: 17050087]

Cramm JM, Nieboer AP. Social cohesion and belonging predict the well-being of community-dwelling older people. BMC Geriatrics. 2015; 15:30-40. [PubMed: 25879773] 
Dean A, Kolody B, Wood P, Matt GE. The influence of living alone on depression in elderly persons. Journal of Aging \& Health. 1992; 4:3-18.

Diez-Roux AV. Neighborhoods and health: where are we and where do we go from here? Rev Epidemiol Sante Publique. 2007; 55:13-21. [PubMed: 17320330]

Diez Roux AV, Borrell LN, Haan M, Jackson SA, Schultz R. Neighbourhood environments and mortality in an elderly cohort: Results from the cardiovascular health study. Journal of Epidemiology and Community Health. 2004; 58:917-923. [PubMed: 15483307]

Elliott J, Gale CR, Parsons S, Kuh D. Neighbourhood cohesions and mental wellbeing among older adults: A mixed methods approach. Social Science \& Medicine. 2014; 107:44-51. [PubMed: 24602970]

Fukunaga R, Abe Y, Nakagawa Y, Koyama A, Fujise N, Ikeda M. Living alone is associated with depression among the elderly in a rural community in Japan. Psychogeriatrics. 2012; 12(3):179_ 185. [PubMed: 22994616]

Holt-Lunstad J, Smith TB, Baker M, Harris T, Stephenson D. Loneliness and social isolation as risk factors for mortality: A meta-analytic review. Perspectives on Psychological Science. 2015; 10:227-237. [PubMed: 25910392]

Kim K, Lee M. Depressive symptoms of older adults living alone: The role of community characteristics. International Journal of Aging \& Human Development. 2015; 80:248-263. [PubMed: 26195500]

Kim HK, McKenry PC. The relationship between marriage and psychological well-Being: A longitudinal analysis. Journal of Family Issues. 2002; 23:885-911.

Kroenke K, Strine TW, Spitzer RL, Williams JB, Berry JT, Mokdad AH. The PHQ-8 as a measure of current depression in the general population. Journal of Affective Disorders. 2009; 114:163-173. [PubMed: 18752852]

Lawton MP, Nahemow L, Yeh TM. Neighborhood environment and the wellbeing of older tenants in planned housing. The International Journal of Aging and Human Development. 1980; 11:211-227. [PubMed: 7203663]

La Gory M, Fitzpatrick K. The effects of environmental context on elderly depression. Journal of Aging and Health. 1992; 4:459-479.

Macintyre S, Maciver S, Sooman A. Area, class, and health: Should we be focusing on places or people? Journal of Social Policy. 1993; 22:213-234.

Ortman, JM., Velkoff, VA., Hogan, H. An aging nation: The older population in the United States, Current Population Reports, P25-1140. U.S. Census Bureau; Washington, DC: 2014.

Robinette JW, Charles ST, Mogle JA, Almeida DM. Neighborhood cohesion and daily well-being: Results from a diary study. Social Science \& Medicine. 2013; 96:174-182. [PubMed: 24034965]

Roh S, Jang Y, Chiriboga DA, Kwag KH, Cho S, Bernstein K. Perceived neighborhood environment affecting physical and mental health: A study with Korean American older adults in New York City. Journal Immigration Health. 2011; 13:1005-1012.

Sampson RJ, Raudenbush SW, Earls R. Neighborhoods and violent crime: A multilevel study of collective efficacy. Science. 1997; 277:918-924. [PubMed: 9252316]

Truong KD, Ma S. A systematic review of relations between neighborhoods and mental health. Journal of Mental Health Policy and Economics. 2006; 9:137-154. [PubMed: 17031019]

Weden MM, Carpiano RM, Robert SA. Subjective and objective neighborhood characteristics and adult health. Social Science \& Medicine. 2008; 6:1256-1270.

Xiu-Ying H, Qian C, Xiao-Dong P, Xue-Mei Z, Chang-Quan H. Living arrangements and risk for late life depression: A meta-analysis of published literature. International Journal of Psychiatry in Medicine. 2012; 43:19-34. [PubMed: 22641928]

Yen IH, Michael YL, Perdue L. Neighborhood environment in studies of health of older adults: A systematic review. American Journal Preventive Medicine. 2009; 37:455-463. 


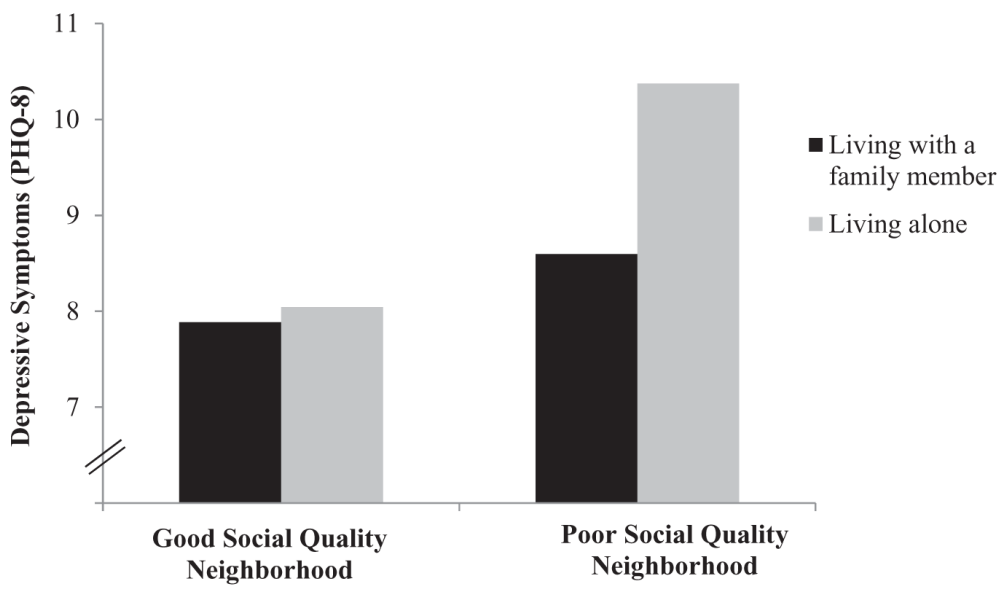

Figure 1.

Effects of residential status and neighborhood social quality on depressive symptomatology. Note: Good/poor social quality of the neighborhood is defined as $1 \mathrm{SD} \pm$ the centered mean $(0)$. 


\section{Table 1}

Participant characteristics by residential status for 1043 adults in Allegheny County, PA, USA, 2014.

\begin{tabular}{lcccc}
\hline Model variables & $\begin{array}{c}\text { Total cohort } \\
(\boldsymbol{N}=\mathbf{1 0 4 3})\end{array}$ & $\begin{array}{c}\text { Living with a family } \\
\text { member }(\boldsymbol{n}=\mathbf{7 3 2})\end{array}$ & $\begin{array}{c}\text { Living alone } \\
(\boldsymbol{n}=\mathbf{3 1 1})\end{array}$ & $\boldsymbol{p}$-value \\
\hline Demographics & & & & \\
$\quad$ Age (years) & $69.17 \pm 9.74$ & $67.33 \pm 8.84$ & $73.46 \pm 10.39$ & $<0.001$ \\
$\quad$ Men, $n(\%)$ & $456(43.6)$ & $341(46.6)$ & $115(37.0)$ & $<0.05$ \\
$\quad$ Black/other, $n(\%)$ & $110(10.6)$ & $61(8.3)$ & $49(15.8)$ & $<0.001$ \\
$\quad$ Bachelor's degree or higher, $n(\%)$ & $289(27.6)$ & $215(29.3)$ & $72(23.3)$ & 0.12 \\
Disability $^{a}$ & & & & \\
$\quad$ ADLs, $n(\%)$ & $27(2.6)$ & $20(2.7)$ & $7(2.2)$ & 0.66 \\
$\quad$ IADLs, $n(\%)$ & $119(11.4)$ & $67(9.1)$ & $52(16.6)$ & $<0.05$ \\
Neighborhood characteristics ${ }^{b}$ & & & & \\
$\quad$ Physical quality & $1.70 \pm 0.52$ & $1.66 \pm 0.51$ & $1.79 \pm 0.54$ & $<0.01$ \\
$\quad$ Social quality & $1.91 \pm 0.63$ & $1.91 \pm 0.59$ & $1.93 \pm 0.63$ & 0.71 \\
Depressive symptoms (PHQ-8) & & & & \\
\hline
\end{tabular}

${ }^{a}$ Number (\%) of participants reporting any ADL/IADL difficulties versus none.

$b_{\text {Lower scores }}=$ better perception of neighborhood.

$c_{\text {Higher scores }}=$ greater frequency of depressive symptoms. 


\section{Table 2}

Participant characteristics by marital status group.

\begin{tabular}{|c|c|c|c|c|c|}
\hline \multirow[b]{2}{*}{ Model variables } & \multirow{2}{*}{$\begin{array}{l}\text { Married/living with a family } \\
\text { member (referent; } n=740 \text { ) }\end{array}$} & \multicolumn{4}{|c|}{ Living alone $(n=303)$} \\
\hline & & Single (69) & Divorced (77) & Widowed (157) & $p$-value \\
\hline \multicolumn{6}{|l|}{ Demographics } \\
\hline Age (years) & $66.68 \pm 8.45$ & $67.56 \pm 9.88$ & $68.06 \pm 8.59$ & $79.06 \pm 8.43$ & $<0.001$ \\
\hline Men, $n(\%)$ & $289(54.1)$ & $37(53.9)$ & $18(23.8)$ & $54(34.3)$ & $<0.05$ \\
\hline Black/other, $n(\%)$ & $20(3.7)$ & $13(18.3)$ & $17(22.0)$ & $19(12.0)$ & $<0.001$ \\
\hline Bachelor's degree or higher, $n(\%)$ & $168(31.5)$ & $24(34.9)$ & $22(29.1)$ & $20(12.8)$ & 0.12 \\
\hline \multicolumn{6}{|l|}{ Disability $^{a}$} \\
\hline ADLs, $n(\%)$ & $14(2.7)$ & $1(2.0)$ & $2(3.2)$ & $3(1.9)$ & 0.60 \\
\hline IADLs, $n(\%)$ & $42(7.9)$ & $12(17.9)$ & $11(13.7)$ & $29(18.3)$ & 0.73 \\
\hline \multicolumn{6}{|l|}{ Neighborhood characteristics $b$} \\
\hline Physical quality & $1.61 \pm 0.48$ & $1.89 \pm 0.50$ & $1.81 \pm 0.59$ & $1.77 \pm 0.53$ & $<0.001$ \\
\hline Social quality & $1.87 \pm 0.57$ & $2.21 \pm 0.65$ & $1.94 \pm 0.72$ & $1.83 \pm 0.54$ & $<0.05$ \\
\hline Depressive symptoms (PHQ-8) ${ }^{c}$ & $3.11 \pm 3.59$ & $5.09 \pm 5.11$ & $4.89 \pm 5.13$ & $3.67 \pm 3.91$ & $<0.001$ \\
\hline
\end{tabular}

${ }^{a}$ Number (\%) of participants reporting any ADL/IADL difficulties versus none.

$b_{\text {Lower scores }}=$ more positive perception of neighborhood.

$c_{\text {Higher scores }}=$ greater frequency of depressive symptoms. 
Table 3

Multiple regression models examining the relations of residential status and neighborhood characteristics on depressive symptomatology.

\begin{tabular}{|c|c|c|c|c|c|}
\hline Variables & $\boldsymbol{B}$ & SE $(B)$ & $t$-stat & $p$ & $R^{2}$ \\
\hline \multicolumn{6}{|l|}{ Demographics } \\
\hline Age & -0.08 & 0.02 & -3.96 & $<0.001$ & \\
\hline $\operatorname{Male}^{a}$ & -0.13 & 0.38 & -0.33 & 0.74 & \\
\hline Black/other ${ }^{b}$ & 0.13 & 0.50 & 0.26 & 0.79 & \\
\hline Education & -0.26 & 0.08 & -3.09 & $<0.01$ & \\
\hline \multicolumn{6}{|l|}{ Disability } \\
\hline ADLs & 0.66 & 0.72 & 0.93 & 0.35 & \\
\hline IADLs & 2.14 & 0.55 & 3.87 & $<0.001$ & \\
\hline \multicolumn{6}{|l|}{ Neighborhood characteristics } \\
\hline Physical quality & 0.56 & 0.38 & 1.50 & 0.13 & \\
\hline Social quality & 1.02 & 0.37 & 2.76 & $<0.01$ & \\
\hline Living alone ${ }^{c}$ & 1.00 & 0.40 & 2.50 & $<0.05$ & \\
\hline Step $1, F(9,982)=12.94 p<0.001$ & & & & & 0.15 \\
\hline \multicolumn{6}{|l|}{ Demographics } \\
\hline Age & -0.07 & 0.02 & -3.81 & $<0.001$ & \\
\hline Male $^{a}$ & -0.20 & 0.38 & -0.52 & 0.60 & \\
\hline Black/other $b$ & 0.05 & 0.49 & 0.11 & 0.91 & \\
\hline Education & -0.24 & 0.08 & -2.89 & $<0.01$ & \\
\hline \multicolumn{6}{|l|}{ Disability } \\
\hline ADLs & 0.69 & 0.76 & 0.91 & 0.36 & \\
\hline IADLs & 2.14 & 0.55 & 3.91 & $<0.001$ & \\
\hline \multicolumn{6}{|l|}{ Neighborhood characteristics } \\
\hline Physical quality & 0.58 & 0.38 & 1.55 & 0.12 & \\
\hline Social quality & 0.59 & 0.46 & 1.29 & 0.20 & \\
\hline Living alone ${ }^{c}$ & -1.61 & 1.23 & -1.31 & 0.19 & \\
\hline \multicolumn{6}{|l|}{ Interactions } \\
\hline $\begin{array}{l}\text { Living alone } \times \\
\text { neighborhood social quality }\end{array}$ & 1.35 & 0.62 & 2.19 & $<0.05$ & \\
\hline Step $2, F(10,981)=11.99, p<0.001$ & & & & & 0.16 \\
\hline \multicolumn{6}{|l|}{ References levels: } \\
\hline \multicolumn{6}{|l|}{$a_{\text {female; }}$} \\
\hline$b_{\text {White; }}$ & & & & & \\
\hline
\end{tabular}

